\title{
Comprehensive processing of high-throughput small RNA sequencing data including quality checking, normalization, and differential expression analysis using the UEA sRNA Workbench
}

\author{
MATTHEW BECKERS, ${ }^{1,3}$ IRINA MOHORIANU, ${ }^{1,2,3}$ MATTHEW STOCKS, ${ }^{1,3}$ CHRISTOPHER APPLEGATE, ${ }^{1}$ \\ TAMAS DALMAY, ${ }^{2}$ and VINCENT MOULTON ${ }^{1}$ \\ ${ }^{1}$ School of Computing Sciences, University of East Anglia, Norwich, NR4 7TJ, United Kingdom \\ ${ }^{2}$ School of Biological Sciences, University of East Anglia, Norwich, NR4 7TJ, United Kingdom
}

\begin{abstract}
Recently, high-throughput sequencing (HTS) has revealed compelling details about the small RNA (sRNA) population in eukaryotes. These 20 to 25 nt noncoding RNAs can influence gene expression by acting as guides for the sequence-specific regulatory mechanism known as RNA silencing. The increase in sequencing depth and number of samples per project enables a better understanding of the role sRNAs play by facilitating the study of expression patterns. However, the intricacy of the biological hypotheses coupled with a lack of appropriate tools often leads to inadequate mining of the available data and thus, an incomplete description of the biological mechanisms involved. To enable a comprehensive study of differential expression in sRNA data sets, we present a new interactive pipeline that guides researchers through the various stages of data preprocessing and analysis. This includes various tools, some of which we specifically developed for sRNA analysis, for quality checking and normalization of sRNA samples as well as tools for the detection of differentially expressed sRNAs and identification of the resulting expression patterns. The pipeline is available within the UEA sRNA Workbench, a user-friendly software package for the processing of sRNA data sets. We demonstrate the use of the pipeline on a $H$. sapiens data set; additional examples on a $B$. terrestris data set and on an $A$. thaliana data set are described in the Supplemental Information. A comparison with existing approaches is also included, which exemplifies some of the issues that need to be addressed for sRNA analysis and how the new pipeline may be used to do this.
\end{abstract}

Keywords: high-throughput sequencing (HTS); microRNA (miRNA); small RNA (sRNA); UEA sRNA Workbench, quality checking; normalization; differential expression

\section{INTRODUCTION}

RNA silencing is known to play a key role in the fine-tuning of gene expression in eukaryotes (Brodersen and Voinnet 2006).The process is mediated by a set of RNA molecules referred to as small RNAs (sRNAs). Well-known examples of sRNAs include microRNAs (miRNAs) (Bartel 2009; Voinnet 2009) and small interfering RNAs (siRNAs) (Carthew and Sontheimer 2009; Meister 2013). These RNA fragments are excised by Dicer/Dicer-like proteins from double-stranded RNA precursors deriving either from single stranded RNAs with a hairpin-like secondary structure, the miRNAs (Zhu et al. 2013), or long double-stranded RNA created by a polymerase, the siRNAs (Chen 2012). The sRNAs

\footnotetext{
${ }^{3}$ Joint first authors

Corresponding author: v.moulton@uea.ac.uk

Article is online at http://www.rnajournal.org/cgi/doi/10.1261/rna. 059360.116. Freely available online through the RNA Open Access option.
}

target and subsequently silence genes and thus play an important role in gene regulation (Lippman and Martienssen 2004; Omidvar et al. 2015), defense against pathogens (Szittya et al. 2010; Donaszi-Ivanov et al. 2013) and general maintenance of the genome (Molnar et al. 2007; Mohorianu et al. 2011).

For most molecular biology experiments, an important question is how the observed phenotype or inherent differences (e.g., time or organ/tissue series) are reflected in the variation in expression of sRNAs, commonly referred to as differential expression analysis or DE analysis (Mohorianu and Moulton 2010; Garber et al. 2011; Ozsolak and Milos 2011; Xu et al. 2014). Identification of DE sequences consists of several distinct stages: first, the quality of the data is investigated to identify (and potentially exclude) samples

(C) 2017 Beckers et al. This article, published in RNA, is available under a Creative Commons License (Attribution 4.0 International), as described at http://creativecommons.org/licenses/by/4.0/. 
containing artifacts such as overrepresenting biases originating from sequencing inaccuracies (Sorefan et al. 2012; Raabe et al. 2014) or introduced from the handling of the original biological sample. Second, the reads are annotated to determine which categories of sRNAs are present. Finally, the expression levels in the samples are normalized to improve the comparability between samples and, subsequently, to refine the accuracy of DE predictions (McCormick et al. 2011; Dillies et al. 2013).

Bioinformatics methods developed for DE analysis have thus far largely focused on analyzing messenger RNA (mRNA) data, first from microarray experiments and now, more commonly, from RNA-seq (mRNA-seq) data sets (Rapaport et al. 2013; Soneson and Delorenzi 2013). Many of these approaches devised for each stage of a DE analysis are transferable to sRNA data sets (see Table 1). However, there are a number of conceptual differences between sRNA microarrays, which capture a small number of known sequences (mainly miRNAs), and sRNA-seq, which capture a wider variety of known and novel sRNAs (usually, in excess of $100 \mathrm{k}$ unique reads). Similar differences in a number of quantified transcripts are also observed between the output of mRNA-seq experiments and sRNA-seq output. More specifically, for mRNA studies, the expression levels of the reads are aggregated into a gene abundance (Mortazavi et al. 2008), whereas each sRNA sequence contributes individually to the distribution of abundances (McCormick et al. 2011; Studholme 2012). Because of this, the resulting distributions are different both in shape; mRNA-seq abundances have a Gaussian-like distribution whereas sRNA-seq abundances follow an exponential-like distribution, and in number of points; thousands of genes compared to millions of unique sRNAs (Barquist and Vogel 2015). In addition, sRNA-seq data have a higher ratio of noise (random degradation products) to signal (genuine sRNAs); due to the nature of sRNAseq processing the median of sRNA abundances lies within the noise range (Vidal et al. 2013). This implies that existing methodologies for microarrays or mRNA-seq DE analyses are applicable but not always appropriate for sRNA-seq data sets (McCormick et al. 2011; Gupta et al. 2012; Lohse et al. 2012; Vidal et al. 2013). Therefore, it is important to develop tools that address the specific characteristics of sRNA-seq data sets and their analysis to complement those currently used for mRNA-seq analysis.

A common approach for HTS data analysis is to group several tools into a pipeline. As well as providing the ability to tailor pipelines to individual experiments, this enables researchers to configure the distinct stages of the analysis as required (Davis et al. 2013). After the setup is complete the (likely lengthy) procedure can be executed without the need for further input from the user. Currently there are several mRNA-seq pipelines available, such as DESeq/DESeq2 (Anders et al. 2013; Love et al. 2014) or edgeR (Zhou et al. 2014) that can be configured to handle, to some extent, the various stages of a sRNA DE pipeline as well (see Table 1).
However, none of these cover the entire analysis of an sRNA data set.

Here we present a comprehensive, interactive processing pipeline for the analysis of sRNA-seq data sets included as part of the UEA small RNA Workbench (Moxon et al. 2008; Stocks et al. 2012). The pipeline summarizes approaches for quality checking (Mohorianu et al. 2011; Axtell 2013), normalization (Dillies et al. 2013), and identification of expression-derived patterns (Lopez-Gomollon et al. 2012; Mohorianu et al. 2013). To enable the user to compare sRNA-seq libraries and indicate the level of confidence to place on predictions made during downstream analysis, we also provide a series of diagnostic plots used throughout the pipeline to assess the characteristics and overall quality of the samples. Users can also evaluate different normalization methods in order to decide which approach is suitable for their data set. In addition, we present a confidence interval (CI)-based approach (Lopez-Gomollon et al. 2012) to summarize the magnitude and direction of fold changes, for each sRNA. On an $H$. sapiens data set, described in the main text, we demonstrate how this can be extended to multiple comparisons that can be used to group sequences with similar patterns across the whole experiment.

\section{RESULTS}

In this section, we illustrate the features of our pipeline on a publically available data set in H. sapiens, GSE47532 (Barrett et al. 2013; Camps et al. 2014) to highlight its use to identify characteristics and diagnose problems in real data. Additional examples are presented in Supplemental Information 1 (example on a $B$. terrestris data set) and in Supplemental Information 3 (example on an A. thaliana data set). The impact of the number of samples or available memory (RAM) on the runtime is discussed in Supplemental Information 2.

\section{Workflows and implementation details}

The pipeline is part of the existing UEA small RNA Workbench package (Stocks et al. 2012), which provides a user friendly environment designed for all users regardless of computing experience. The latest version of the workbench also facilitates the chaining together of multiple tools within a workflow. This allows each distinct part of a pipeline to be fully configured prior to runtime forgoing the need for many separate programs that require interlinked inputs/outputs. For example, given a set of sRNA samples, a workflow for the identification of DE sRNAs could consist of the quality checking of the samples, the normalization of expression levels, the identification of differentially expressed, annotated reads and the overview of resulting expression patterns-a diagram illustrating this series of steps is presented in Figure 1A. Within the workbench interface, the workflow (Fig. 1C) consists of multiple user configurable nodes that represent the various stages in the analysis. 
TABLE 1. A summary of current tools designed for RNA-seq analysis, which can be applied for sRNA-seq analyses

\begin{tabular}{|c|c|c|c|c|c|c|c|c|c|c|c|}
\hline Tool & Format & Data type & $\begin{array}{l}\text { Fastq } \\
\text { QC }\end{array}$ & $\begin{array}{l}\mathrm{Nt} \\
\text { freq }\end{array}$ & $\begin{array}{l}\text { Adp } \\
\text { trim }\end{array}$ & $\begin{array}{l}\text { Size } \\
\text { class }\end{array}$ & Annotation & $\begin{array}{l}\text { MA/ } \\
\text { scatter }\end{array}$ & Norm & $\mathrm{DE}$ & References \\
\hline DeSeq & R library & RNA-seq & $\mathrm{N}$ & $\mathrm{N}$ & $\mathrm{N}$ & $\mathrm{N}$ & $\mathrm{N}$ & $\mathrm{Y}$ & DEseq & $\mathrm{Y}$ & Love et al. (2014) \\
\hline edgeR & $\mathrm{R}$ library & RNA-seq & $\mathrm{N}$ & $\mathrm{N}$ & $\mathrm{N}$ & $\mathrm{N}$ & $\mathrm{N}$ & $\mathrm{Y}$ & TMM & $\mathrm{Y}$ & Zhou et al. (2014) \\
\hline baySeq & $\mathrm{R}$ library & RNA-seq & $\mathrm{N}$ & $\mathrm{N}$ & $\mathrm{N}$ & $\mathrm{N}$ & $\mathrm{N}$ & $\mathrm{N}$ & Quantile & Y & $\begin{array}{l}\text { Hardcastle and Kelly } \\
\text { (2010) }\end{array}$ \\
\hline RSEQtools & Software & mRNA-seq & $\mathrm{N}$ & $\mathrm{N}$ & $\mathrm{N}$ & $\mathrm{N}$ & $\mathrm{Y}$ & $\mathrm{N}$ & RPKM & $\mathrm{Y}$ & Habegger et al. (2011 \\
\hline DARIO & web & ncRNA-seq & $\mathrm{N}$ & $\mathrm{N}$ & $\mathrm{N}$ & $\mathrm{Y}$ & $\mathrm{Y}$ & $\mathrm{N}$ & - & $\mathrm{N}$ & Fasold et al. (2011) \\
\hline Cyber-T & Web & RNA-seq & $\mathrm{N}$ & $\mathrm{N}$ & $\mathrm{N}$ & $\mathrm{N}$ & $\mathrm{N}$ & $\mathrm{N}$ & Logarithmic, VSN & Y & $\begin{array}{l}\text { Kayala and Baldi } \\
\text { (2012) }\end{array}$ \\
\hline ncPRO-seq & Software & sRNA-seq & $\mathrm{Y}$ & $\mathrm{Y}$ & $\mathrm{N}$ & $\mathrm{Y}$ & $\mathrm{Y}$ & $\mathrm{N}$ & - & $\mathrm{N}$ & Chen 2012 \\
\hline Shortran & Software & sRNA-seq & $\mathrm{N}$ & $\mathrm{N}$ & $\mathrm{Y}$ & $\mathrm{N}$ & $\mathrm{N}$ & $\mathrm{N}$ & Total count & $\mathrm{Y}$ & Gupta et al. 2012 \\
\hline RobiNA & Software & RNA-seq & Y & $\mathrm{Y}$ & Y & $\mathrm{N}$ & $\mathrm{N}$ & $\mathrm{N}$ & RPKM & DeSeq/edgeR & Lohse et al. 2012 \\
\hline omiRas & Web & miRNA-seq & Y & $\mathrm{N}$ & Y & $\mathrm{N}$ & $\mathrm{N}$ & $\mathrm{N}$ & DESeq & DeSeq & Müller et al. (2013) \\
\hline Kraken & Software & RNA-seq & Y & $\mathrm{Y}$ & Y & $\mathrm{N}$ & $\mathrm{N}$ & $\mathrm{N}$ & - & N & Davis et al. 2013 \\
\hline TCC & R library & RNA-seq & $\mathrm{N}$ & $\mathrm{N}$ & $\mathrm{N}$ & $\mathrm{N}$ & $\mathrm{N}$ & $\mathrm{N}$ & DEGES/TbT & Multiple & Sun et al. (2013) \\
\hline sRNAtoolbox & Web & sRNA-seq & $\mathrm{N}$ & $\mathrm{N}$ & $\mathrm{Y}$ & $\mathrm{N}$ & $\mathrm{N}$ & $\mathrm{N}$ & edgeR, NOlseq & edgeR, NOlseq & Rueda et al. (2015) \\
\hline $\begin{array}{l}\text { UEA sRNA } \\
\text { Workbench }\end{array}$ & Software & sRNA-seq & Y & $\mathrm{Y}$ & $\mathrm{Y}$ & Y & $\mathrm{Y}$ & $\mathrm{Y}$ & $\begin{array}{l}\text { RPM, quantile, } \\
\text { subsampling, } \\
\text { DESeq, TMM }\end{array}$ & $\mathrm{Y}$ & Stocks et al. (2012) \\
\hline
\end{tabular}

For each tool we present the type of expected input (e.g., mRNA-seq, sRNA-seq, etc.), the availability of quality checks, analysis of the nucleotide distributions, and possibility of adapter trimming. Additional features include the evaluation of size class distributions and MA or scatter plots. Higher level checks such as the annotation of reads, normalization of abundances, and differential expression calls are also reviewed.

A standard pipeline takes as input sequence data in FASTA format with the adapters trimmed. The files can be generated using the adapter removal tool (Stocks et al. 2012) which also allows users to process samples created using the HD sequencing protocol (Sorefan et al. 2012). The next step is the configuration of the workflow using the setup wizard. The first stage is to organize the data/samples in a manner that reflects the original wet lab experimental design. The sample hierarchy is represented as a tree diagram where leaf nodes represent the replicates and the parents represent the individual samples (Fig. 1B). Users then provide a reference genome and an (optional) GFF file, corresponding to the genome build, which will be used for the annotation stage. If an annotation file is provided, users can then choose which annotations are relevant for the analysis.

After configuring the sample files, users can choose to begin the workflow immediately or enter each stage of the workflow and change the configurable parameters, as necessary. In addition, during the workflow, users can mark problematic replicates (resulting from the first stage of quality checking) or individual size classes for removal, then select up to six normalization methods to be investigated. The quality check reports are then recreated on the normalized data and can be inspected. Next, the user can select the method that best corrects the data artifacts based on the nuanced characteristics of the data set's expression distributions.

The quality check, normalization, and DE steps are computationally intensive and pose significant demands on both processor and in particular memory (RAM). To counteract this issue, we developed a series of back end improvements, which enable users with a wide range of computing hardware to use the pipeline. More specifically, we used disk solutions based on relational database management interfaced with a Java front end and interacted via a JavaScript GUI (which is also used to display resulting graphs and tabular results). However, as the use of disk for runtime storage and calculations can have significant impacts on processing time, a RAM-only version of the software is also available for users with access to high-end computing hardware.

\section{Quality checking}

To illustrate the quality check stage of the pipeline, initial checks on a $H$. sapiens data set ( $\mathrm{H}$ data) were conducted both before and after aligning reads to the reference genome. The first step of the pipeline is to evaluate the overall features of the data being analyzed. The sequencing quality of individual sRNA-seq samples is initially assessed based on the positional nucleotide composition. Next, the total library size (redundant count) and the total number of unique sequences (non-redundant) count are compared across libraries to assess the variation in sequencing depth. The size class distributions for both redundant and non-redundant reads (Fig. 2A1, A2) can indicate abundant or otherwise important sRNA classes early on in the analysis, or identify issues with the sequencing or mapping of certain size classes. The distribution of complexities, defined as the ratio of redundant to nonredundant reads, provides an approximation of the number and abundances of reads in each size class (Fig. 2A3). Complexity values that are close to 1 indicate a highly diverse set of low abundant sequences, whereas lower complexity values are caused by a smaller set of highly abundant 
A

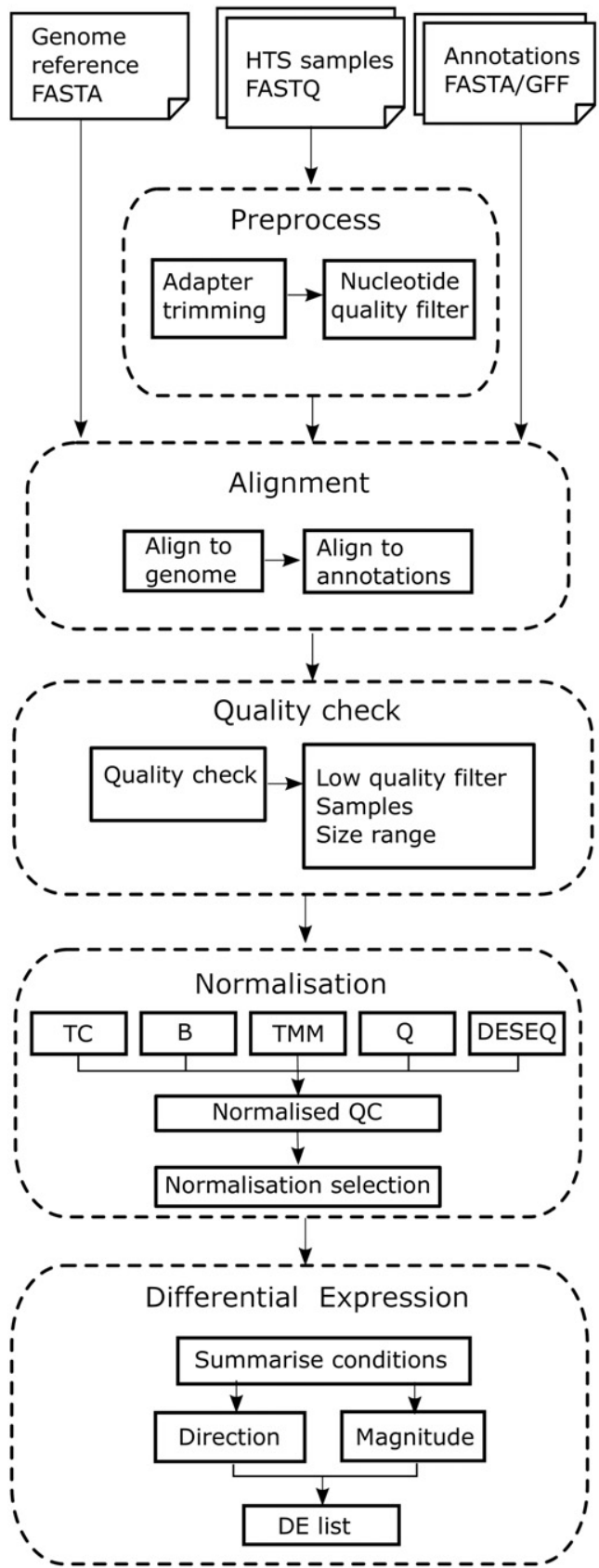

B

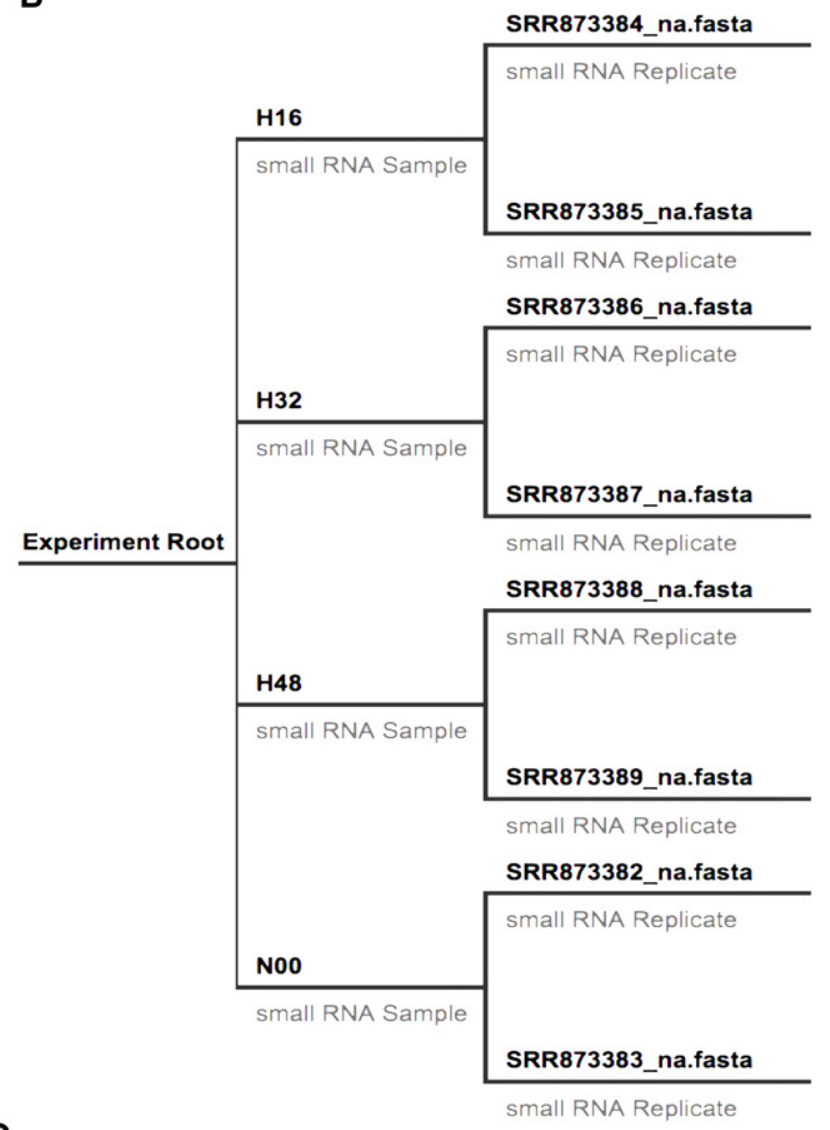

C

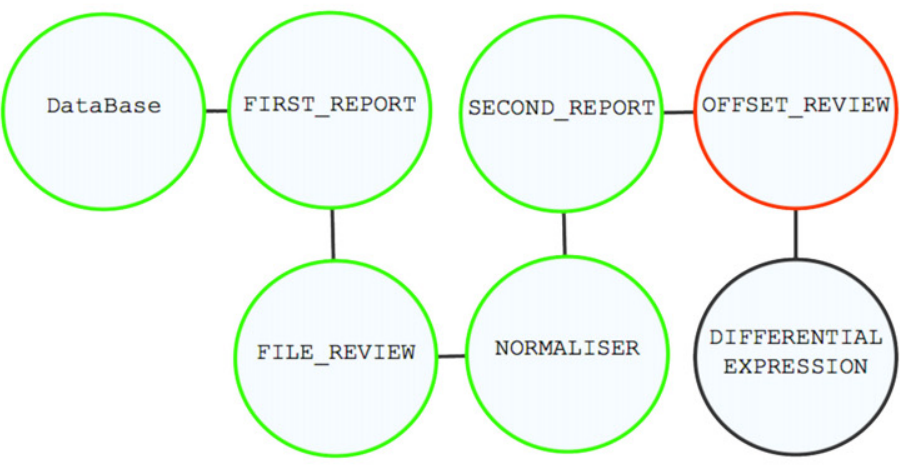

FIGURE 1. Overview of the analysis pipeline and the input of the Differential expression workflow implemented in the UEA sRNA Workbench. (A) Diagram showing the steps of the pipeline, including the preprocessing, alignment to the reference genome and available annotations, quality checking of the raw and processed data, normalization and differential expression call. $(B)$ Hierarchical representation of the input data obtained using the input wizard. $(C)$ The user interface for a workflow containing Quality Checks, Normalization and Differential Expression call; each node can be configured individually.

sequences (Mohorianu et al. 2011). For the $\mathrm{H}$ data set, we observe a peak in the redundant count distribution at 22-23 nt and a sharp and focused decrease in complexity (Figure 2A1, A3). This indicates the presence of a few highly abundant sRNAs for these particular lengths. We also notice that one replicate of the $\mathrm{H} 32$ condition contains more unique reads than the other samples for sizes lower than $22 \mathrm{nt}$, and that there is a markedly higher complexity for an $\mathrm{H} 16$ replicate across the lower and higher range of size classes, indicating an over representation of read variants.

The qualitative replication analysis is conducted through the replicate versus replicate scatter plots and MA plots/ Bland-Altman plots (Bland and Altman 1986), Figure 2B, with similar characteristics and interpretation to those on microarray data (Bolstad et al. 2003; McCormick et al. 2011; Dillies et al. 2013); for the latter each dot corresponds to a 
A.1

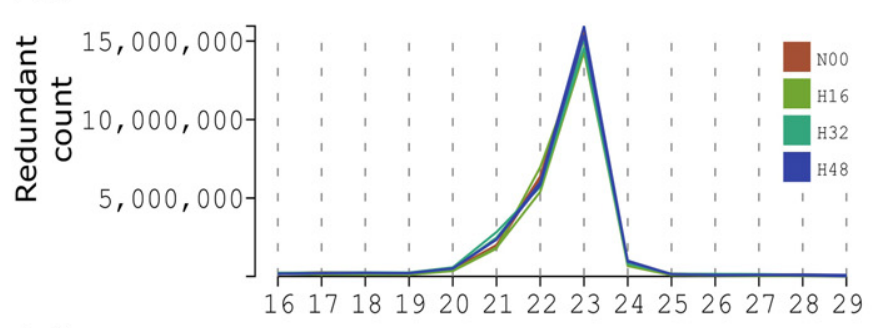

A.2
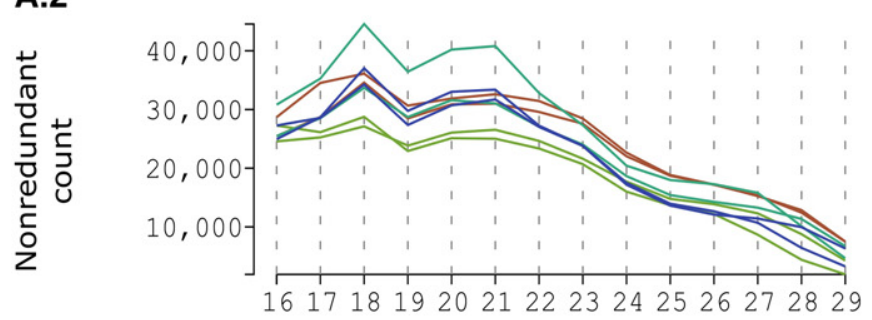

A.3
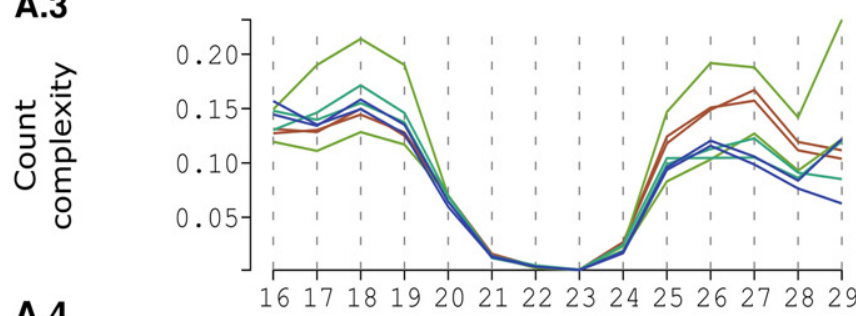

A.4

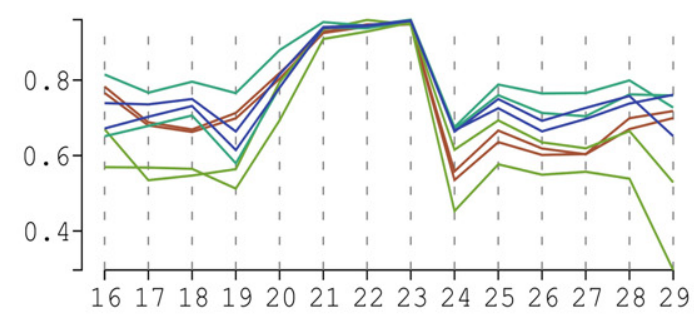

A.5
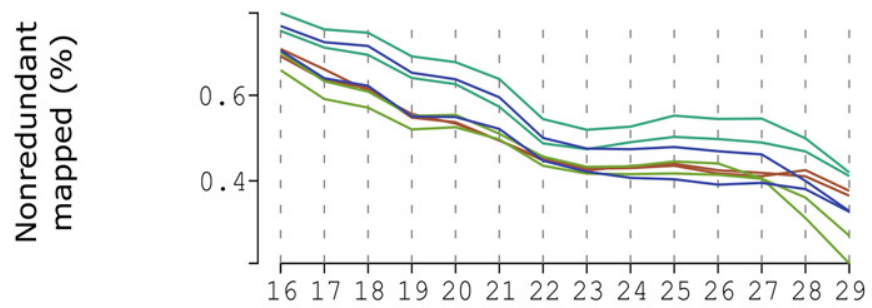

B
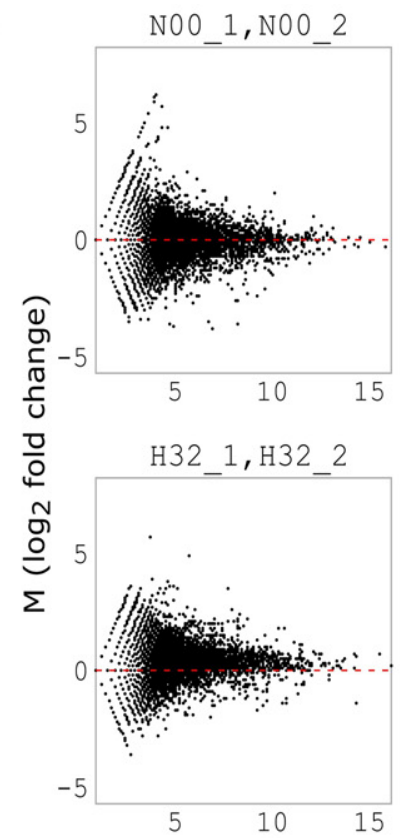

C

A ( $\log _{2}$ average abundance)

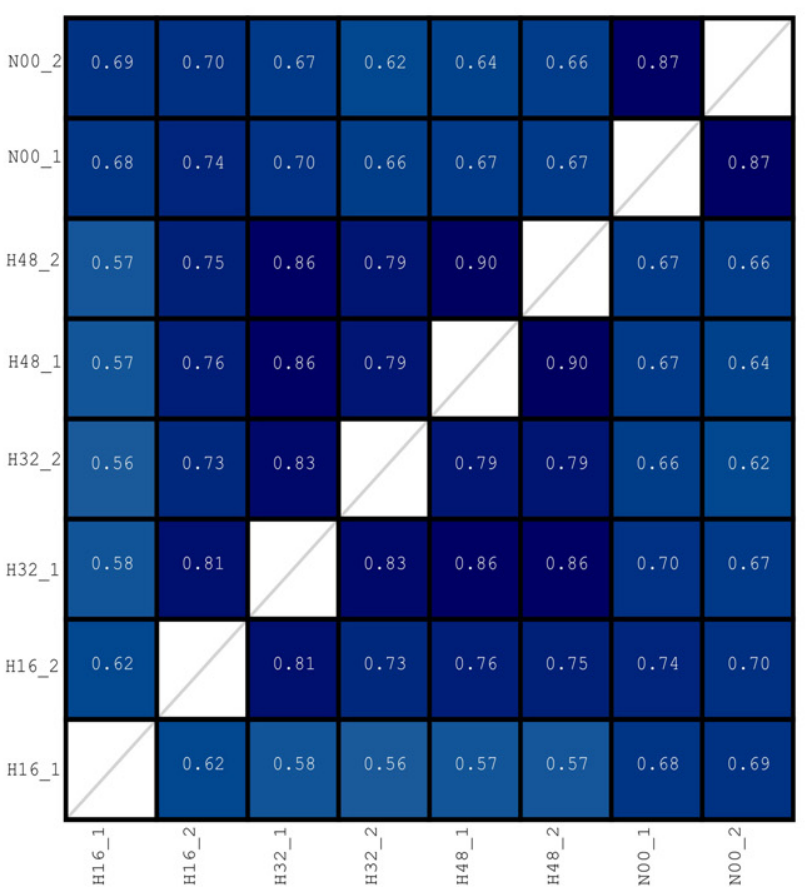

FIGURE 2. Quality checks for the $\mathrm{H}$ data set. $(A)$ The characterization of reads within a sample can be obtained by creating the size class distributions for redundant (A.1) and non-redundant (A.2) reads. Next, the ratio of unique to total reads can be investigated using the complexity distribution (A.3). Lastly, the proportions of genome matching reads for redundant (A.4) and non-redundant (A.5) reads highlight the quality of the sRNA library. (B) MA plots on the raw abundances (prior to any normalization or filtering) for evaluating the reproducibility of the replicates. On the $x$-axis we represent the average abundance between the replicates; on the $y$-axis we represent the fold changes. Good samples show low variability with the increase of abundance (e.g., N00, H32, and H48); problematic samples are characterized by high variability between replicates (e.g., H16). (C) Jaccard similarity indexes computed on the top 1000 most abundant reads. These indicate a high reproducibility between the N00, H32, and $\mathrm{H} 48$ replicates (in excess of 0.8 ) and a low reproducibility for the H16 replicates (0.62). Interestingly, the second H16 replicate is more similar to the first replicate in the H32 time point.

gene, in this context each dot represents an sRNA. This comparative analysis can be extended to higher levels (such as at the sample or treatment level) and it should be reviewed again using the normalized expression levels. For the $\mathrm{H}$ data set, this analysis indicated a high consistency for the $\mathrm{H} 32$ and $\mathrm{H} 48$ replicates and reduced agreement between 
the H16 replicates. Supporting the initial observation, the most dispersed size-separated fold changes are those found between the replicates of H16 (Fig. 2B). Low Jaccard indices generated in the second report indicate that these replicates have poor comparability caused by large differences in both the sequence count distribution and sequence composition of the first replicate (Fig. 2C). Since there are only two replicates per treatment and there is no objective approach for choosing one of the two, this plot indicates that the H16 treatment should probably be excluded from further analysis. The other treatments show a high similarity between replicates, with very few fold changes greater than an absolute $\log _{2}$ fold change of one at higher average expression levels. Although treatment $\mathrm{H} 32$ shows a slight skew toward positive fold changes caused by a higher sequencing depth in the second replicate, the pipeline can be used to correct this issue at the normalization stage.

The percentage of genome-matching reads is also calculated for both redundant and non-redundant sequences and across size classes (Fig. 2A). In addition to examining the entire sRNA population in a data set, all quality checks described so far can also be calculated and compared visually across individual annotations of interest. These include miRNAs, other ncRNAs (such as tRNAs, rRNAs, or snoRNAs), protein-coding genes and repeat/ transposable elements depending on available annotation information (Mortazavi et al. 2008; Xu et al. 2014). These analyses indicate a high proportion of reads in these samples are likely to be miRNAs.

\section{Normalization}

The next step in the pipeline is the normalization of the expression levels. In the normalization node we incorporate several existing methods for normalization, with additional features that we have developed especially for sRNA data sets. For scaling-based methods, the normalization total influences the subsequent DE call; ideally it should not be much lower than the original number of reads. For example, if the scaling is done at $1 \mathrm{M}$ for samples with $>10 \mathrm{M}$ reads then all the expression levels will be reduced and DE may be hidden. Alternatively, if scaling is done at $10 \mathrm{M}$ for samples with $<1 \mathrm{M}$ reads, then DE could be artificially be generated. An appropriate normalization total therefore lies in the same range as the sample totals (the average and median options are presented as alternatives). Other options are rank-based quantile normalization adapted for sRNA-seq data (Bolstad et al. 2003) and subsampling normalization (Li et al. 2012).

The analysis of the $\mathrm{H}$ data set highlights a common issue with normalization where two replicates are sequenced with different overall depths (mainly due to the characteristics of the sequencing platform used). To evaluate which method(s) are suitable for this data set, we tested all six normalization techniques described in Materials and Methods. Figure 3 illustrates the size-separated distributions of differential expression which can be used to evaluate the suitability of each normalization method. Fold-changes between replicates should be minimal and produce a distribution centered on zero, after normalization. While the TMM, DESeq2, and quantile methods appear to center the distributions of all size classes, the total count, subsampling, and upper quartile methods do not improve on the comparability of the distributions. This suggests that for the $\mathrm{H}$ data, either TMM, DESeq, or quantile normalization should be chosen as normalization approaches.

\section{Differential expression comparison with existing approaches}

We exemplify the DE analysis on the $\mathrm{H}$ data set for two comparisons: N00/H32 and H32/H48; the left-hand side is considered the reference sample.

We compared our results, obtained using the offset fold change, in $\log _{2}$ scale (LOFC) and confidence interval (CI) pattern approach-described in Materials and Methods, with two of the most widely used tools for detecting DE reads, DESeq2 (Love et al. 2014), and edgeR (Zhou et al. 2014). Both approaches control for false positives by estimating dispersions and weighting fold changes based on these dispersion estimates. For the DESeq 2 and edgeR analyses we used a significance cut-off of 0.05 . For the method implemented in the workbench, we applied a threshold of 1 LOFC (both for $U$ and D patterns) to call sequences as DE. This was selected based on empirical evidence that a sequence with a $\log _{2}$ fold change of one can be detectable on a Northern blot or via qPCR (Morey et al. 2006). The KL divergence curves generated from the $\mathrm{H}$ data set used for determining the appropriate offsets are shown in Figure 4. We also assessed the dependence of the offset on the number of strand bias bins and length of the alignment window. In the $\mathrm{H}$ data set, the number of strand bias bins heavily affected the resulting offset up to 100 bins, after which point the KL curve remains unchanged, which resulted in an offset biased toward the lower end of abundance levels. The offset was also affected by alignment window length and can vary erratically when using the raw measures; however, we utilize a LOESS smoothing function (Cleaveland 1979) to produce a more stable offset.

For the N00 versus H32 comparison of the H data set, 427 sequences were called DE by all methods (Fig. 5B). DESeq2 and edge $\mathrm{R}$ both predicted 241 sequences not called DE by the LOFC method; DESeq 2 returned 110 differentially expressed sequences that edgeR and our method did not find significant, and edgeR predicted 15 differentially expressed sequences which were not captured using the other methods. Based on the MA plot (Fig. 5A) we observe that the abundance and/or offset fold-change of these specific calls is low. These artifacts can be identified and evaluated on a case-by-case basis by using the LOFC and the CI approach. In addition, we present the expression levels of the four reads identified exclusively using the LOFC approach (Fig. 5C). 


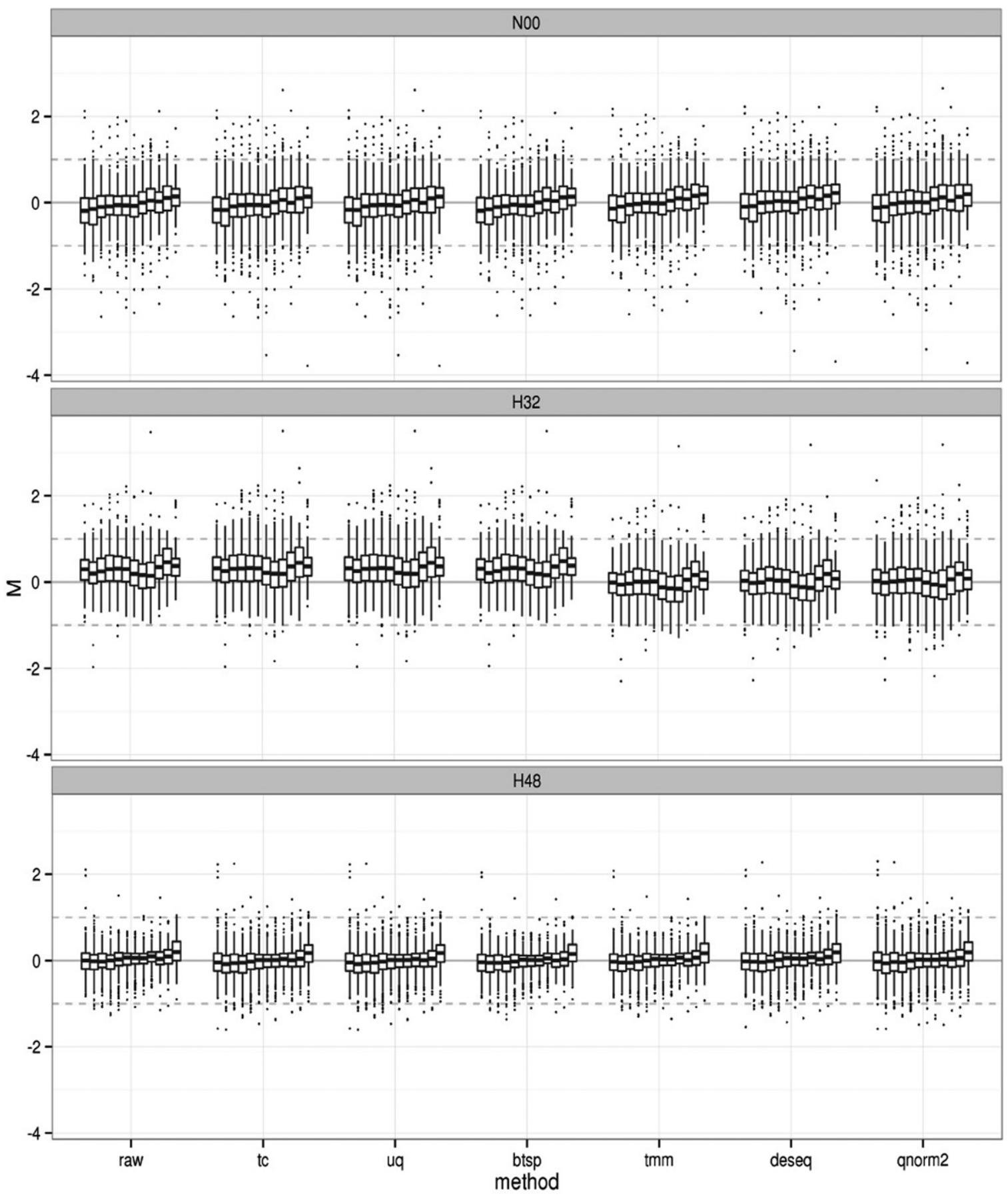

FIGURE 3. Evaluation of the appropriateness of the normalization methods on the $\mathrm{H}$ data set. For each sample and for each set of replicates, we represent the fold-change distributions ( $y$-axis) for each individual size class ( $x$-axis). Based on the assumption that no significant differences are expected between replicates, a suitable normalization is one that brings all distributions on the 0 line (in $\log _{2}$ scale, this corresponds to equal values in both replicates). For the $\mathrm{H}$ data set, the TMM, DeSeq2, and adapted quantile normalization fulfill this criterion for all samples.

\section{DISCUSSION}

We have described a sRNA processing pipeline, part of the UEA sRNA Workbench, that includes steps for quality checking, normalization, and identification of DE sRNAs considering the unique characteristics of sRNA-seq data sets.
To achieve a better understanding of these data sets, the pipeline generates a set of diagnostic plots, which can be used initially to review the raw sequencing quality of the replicates and then to assess the effect that different normalization techniques have of the abundance distributions. The use of a suitable normalization is essential for reducing false-positive 


\section{N00_1}

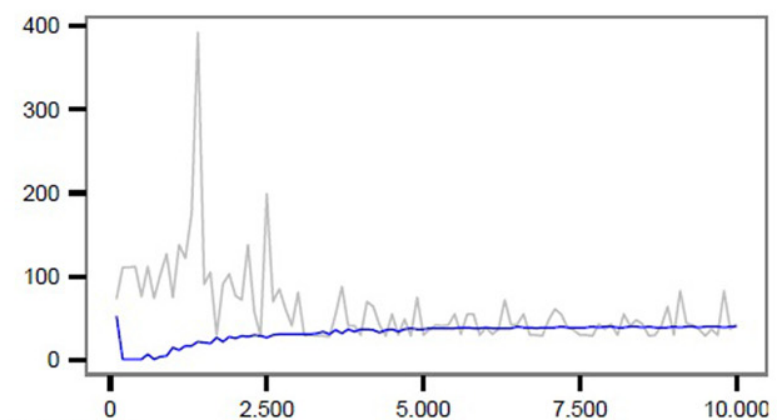

H32_1

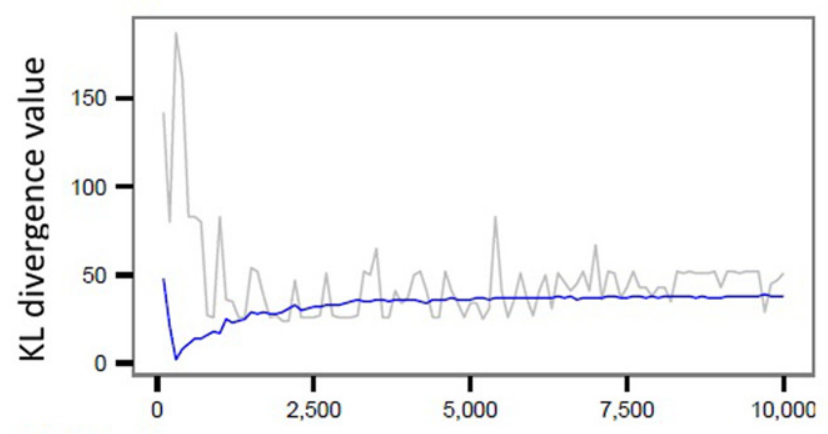

H48_1

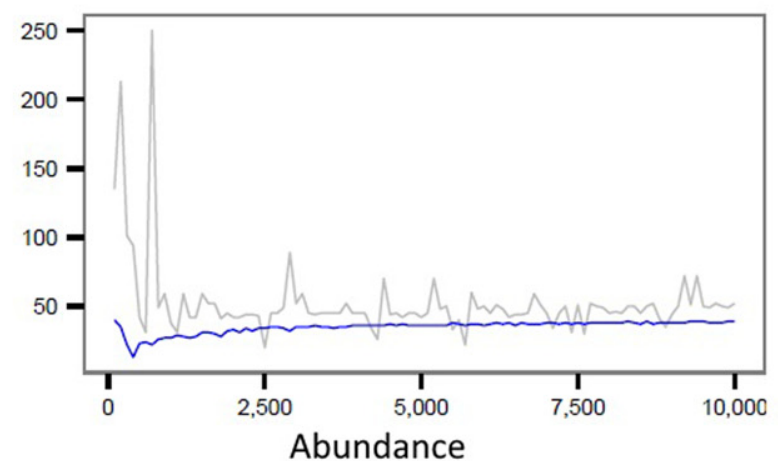

N00_2

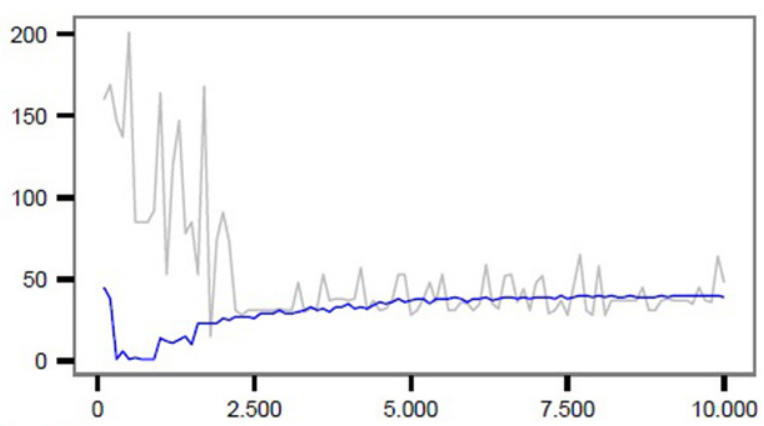

H32_2

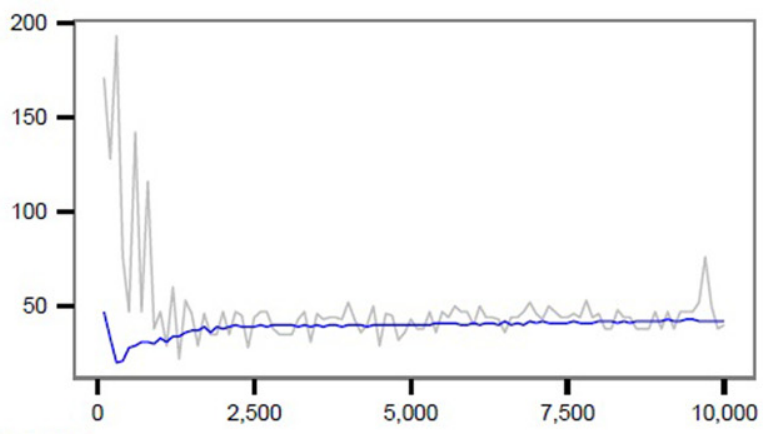

H48_2

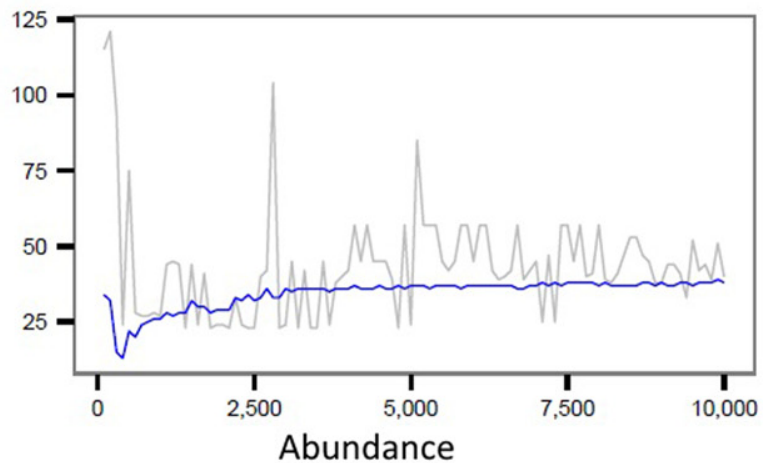

FIGURE 4. Identification of an offset for each sample in the $\mathrm{H}$ data set using Kullback-Leibler divergence to compare the strand bias distributions of reads to a random uniform distribution, in windows of various lengths. This analysis was done on windows of length $1000 \mathrm{nt}$ (parameter that can be modified from the GUI), for each of the two replicates (_1 and _2) of the three accepted samples, N00, H32, and H48. On the $x$-axis we show the abundances of the considered windows (the abundance within a window is the algebraic sum of the abundances of all incident reads); on the $y$-axis we represent the value of the KL divergence. The gray line indicates the unsmoothed KL divergence values and the blue line shows the divergence values smoothed by LOESS ( span $=0.3$ ). The offset for each sample is determined as the minimum of the smoothed divergence. The offset for the whole data set is the overall minimum of these values; for this data set this value was determined to be 42 .

predictions; however, no single normalization technique can be invariably applied to all sRNA-seq data sets. To evaluate which approach is appropriate for a given data set (i.e., by rendering the samples comparable from most [preferably all] quality check angles) we encourage the user to investigate their using the revised quality check plots.

When identifying DE transcripts in HTS data it is important to take into account the level of noise, a quantity that increases with the depth of sequencing. To account for this, we have implemented a user-friendly tool for the iden- tification of a suitable offset, which estimates the abundance range of the reads lacking sRNA characteristics (e.g., specific size), taking into account the sequencing depth and the characteristics of the sRNA population present in the samples. We compared the results of our DE analysis (LOFC) to that of DESeq2 and edgeR DE packages to determine the level of overlap between other methods and our own. In lieu of a $P$-value threshold to assess DE genes, which often reports large numbers of significant genes often with a low difference in expression, we used a cut-off of 1 LOFC to 

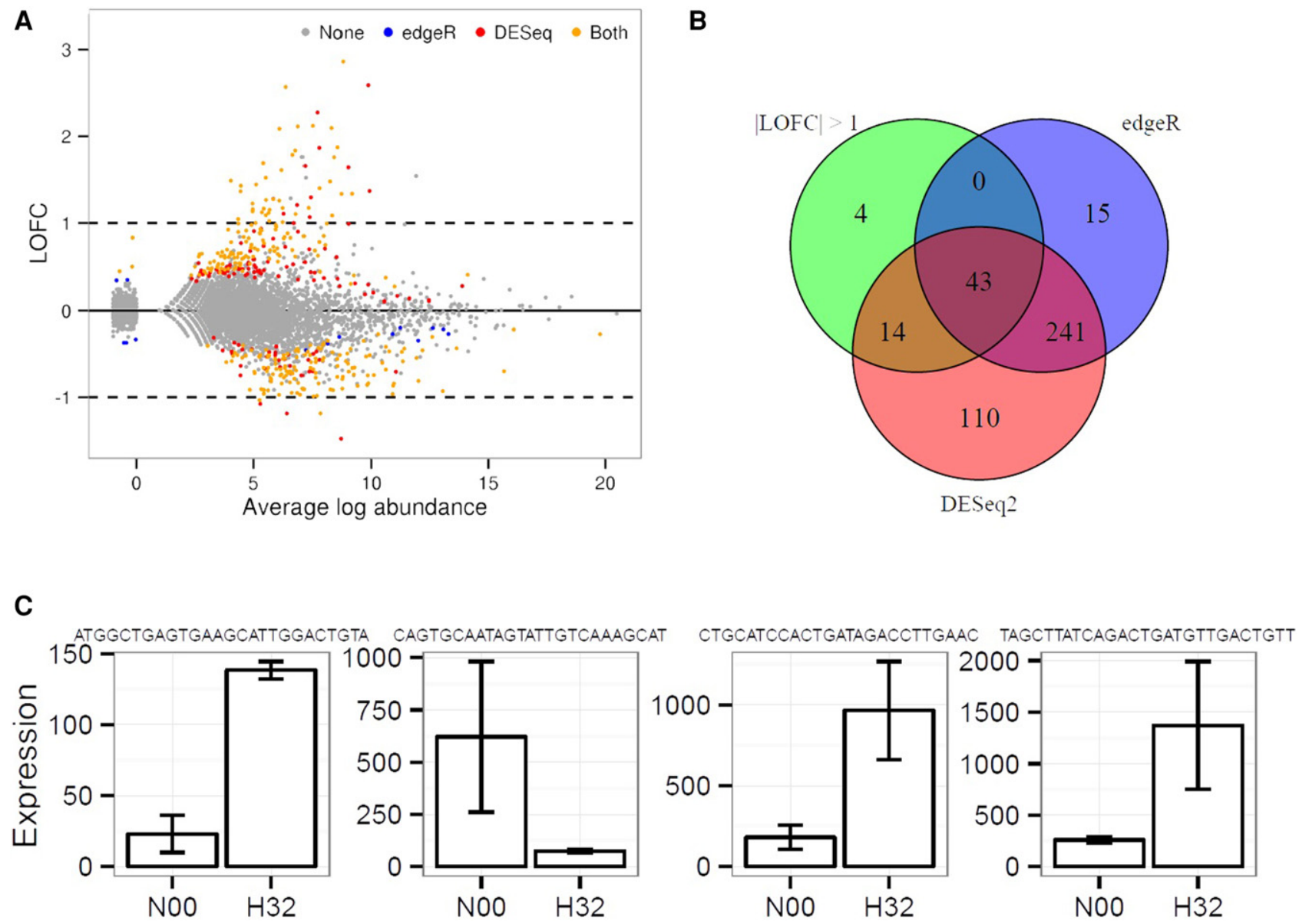

FIGURE 5. Assessment of three approaches used for the identification of differentially expressed reads applied on the N00 versus $\mathrm{H} 32$ comparison ( $\mathrm{H}$ data set). (A) MA plot created using the normalized expression levels (TMM method, see Fig. 3). On the $x$-axis we represent the average abundance; on the $y$-axis we represent the $\log _{2}(\mathrm{OFC})$. The color of the dots indicates whether the reads were called DE by both edgeR and DESeq2 (orange), exclusively by edgeR (blue), or exclusively by DESeq2 (red). Reads accepted as DE using the LOFC approach are those outside the dotted lines. (B) Venn diagram showing the number of reads called DE using the LOFC, edgeR, and DESeq2 methods. $(C)$ Distributions of expression levels (represented as maximal intervals) for the four sequences called DE exclusively by the LOFC method.

filter the reported sequences. The cut-off can, however, be user defined in order to reduce or increase the number of reported sequences. Importantly, the ranking of sequences by LOFC is not populated with high but insignificant fold changes.

To further accommodate the variability between replicates we use CIs created over normalized replicate expression levels which produce more stringent lists of DE sequences between treatments. The method is also extended to multiple conditions by using pattern-based grouping of the sequences (Fig 6). The method is not only suitable for (ordered) time-series data sets, but can also be applied to other types of comparative experiments such as wild-type versus multiple treatments or cross tissue comparison. Grouping DE sequences allows users to quickly view sets of sRNAs that follow the same pattern of expression throughout the experiment.

During our analyses we observed that problematic data sets arise when whole size classes are affected by a condition, causing a high rate of $\mathrm{DE}$ for a large proportion of the
sRNAs, e.g., RNAi mutants which cause the exclusion of a whole class of sRNAs or virus infections which produce a large set of viral siRNAs in the infected samples (Szittya et al. 2010). To our knowledge no current normalization is able to correct for such experiments, and further approaches will need to be developed to provide an appropriate normalization solution to this kind of data.

In conclusion, we have described a user-friendly pipeline for sRNA DE analysis that allows the evaluation of a variety of techniques to identify the most suitable approach for a given data set. The workbench includes both established approaches and tools that we have specifically developed for sRNA sequence analysis and facilitates a coherent and informed analysis through linking the different aspects into a workflow. The UEA sRNA Workbench and the pipeline design devised for the data analysis may prove to be a valuable resource facilitating the expansion of our knowledge of sRNAs, especially for the study of new or less well characterized classes of sRNAs. 


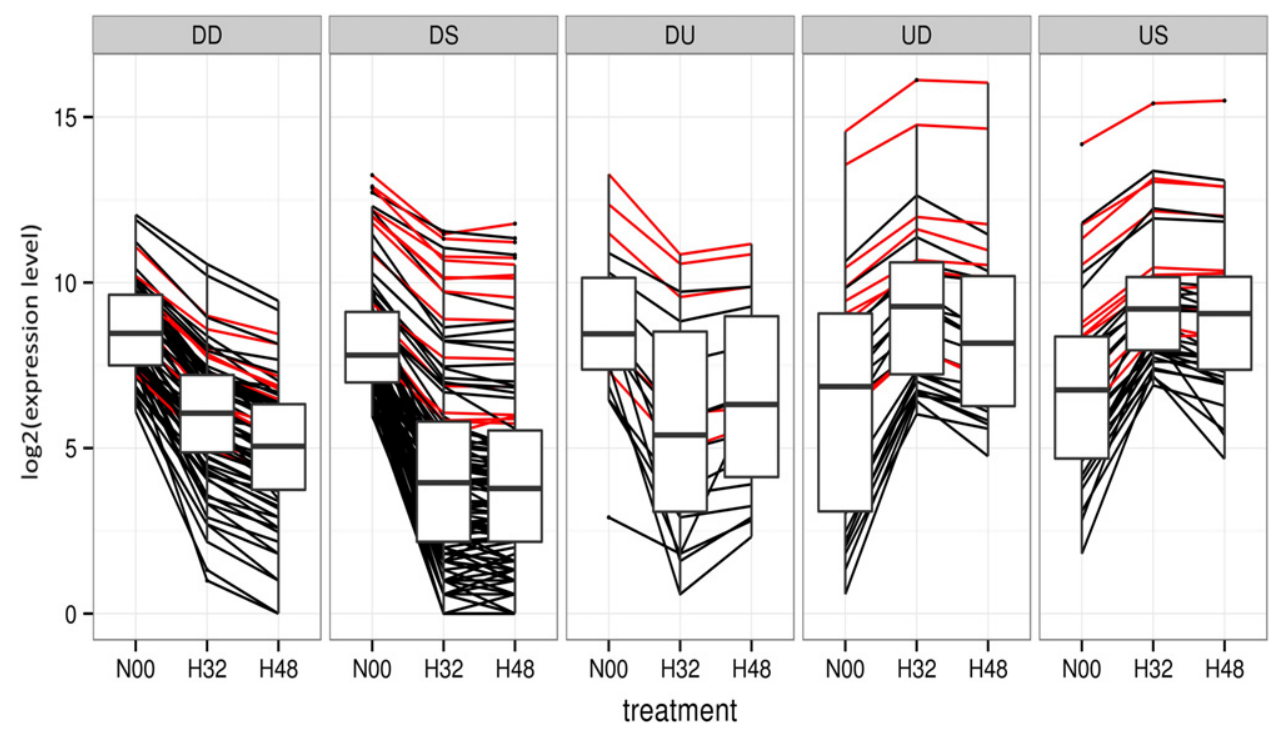

FIGURE 6. Clusters of reads sharing similar patterns (only the clusters with more than 15 entries were presented; the SS cluster was excluded, since the vast majority of the reads are not expected to be differentially expressed between treatments). The $U$ and $D$ descriptors were assigned to reads for which the LOFC on the proximal ends of the maximal expression intervals was in excess of one. Each line corresponds to the averaged expression profile, on the two available replicates, for one sRNA; the red lines are used to highlight miRNA expression profiles. The boxplot interquartile ranges (IQRs) are used to highlight the distributions of expression in each time point and underline the pattern.

\section{MATERIALS AND METHODS}

To illustrate the use of the pipeline we use a $H$. sapiens data set referred to as " $\mathrm{H}$ " data (publicly available on Gene Expression Omnibus [GEO] under accession number GSE47532). This is an experiment on the effects of hypoxic conditions on MCF7 cells (Camps et al. 2014), organized into a time series of four points, each with two biological replicates, Normoxia (N00), Hypoxia at $16 \mathrm{~h}(\mathrm{H} 16)$, Hypoxia at $32 \mathrm{~h}$ (H32), and Hypoxia at $48 \mathrm{~h}$ (H48). The additional examples presented in the Supplemental Information are based on publicly available $B$. terrestris data (GSE64512) consisting of two samples, with four biological replicates each (Sadd et al. 2015) and a publicly available $A$. thaliana data (GSE35562, GSM1178880 to GSM1178882 for the wild-type and GSM1178883 to GSM1178885 for the Hen 1-8 mutant) consisting of two samples, with three biological replicates each (Zhai et al. 2013).

In this section, we describe the methodology and software underpinning the new pipeline; the main workflow diagram is presented in the diagram in Figure 2A.

\section{Quality checking}

The sequencing quality of individual sRNA-seq samples is assessed based on properties such as the positional nucleotide composition (SEQC/MAQC-III Consortium 2014), sequencing depth and the number of unique sequences present in a sample (Rajagopalan et al. 2006). The accuracy of expression replication is evaluated by comparing, qualitatively and quantitatively, the abundances of reads between replicates (Mapleson et al. 2014). The quantitative analysis includes the study of size-class separated distributions of abundances and complexities, defined as the ratio of unique (non-redundant) to total (redundant) reads and the Jaccard similarity index on the top 500 most abundant reads (Jaccard 1901; Mohorianu et al. 2011). The qualitative comparison is conducted through the repli- cate versus replicate scatter plots and MA plots/Bland-Altman plots (Bland and Altman 1986). We also assess the stability of distributions of fold changes between replicate libraries for each size class presented in $\log _{2}$ scale. An appropriate similarity between the compared replicates/samples is indicated by tight distributions, symmetric on $0 \log _{2}$ fold change with no deviations for any particular size classes (Yang et al. 2002; Mohorianu et al. 2011). The percentage of genome-matching reads is calculated for both redundant and non-redundant sequences and across size classes. Selected annotations for which similar checks are performed include miRNAs, other ncRNAs (such as tRNAs, rRNAs, or snoRNAs), protein-coding genes, and repeat/transposable elements depending on available annotation information (Xu et al. 2014; Omidvar et al. 2015).

Abundance distributions of reads in each sample are plotted in a series of boxplots (McCormick et al. 2011; Dillies et al. 2013). However, due to the high proportion of low abundance reads characteristic to sRNA-seq data these distributions for all reads are often uninformative. To counter this, we break the data into abundance ranges of user-defined length (referred to as abundance windows) and assess the comparability of the sample distributions within each window.

\section{Normalization}

The aim of the normalization of the expression levels is to minimize the technical variation between replicates and treatments which is not biologically relevant, e.g., sequencing errors and biases or artifacts from the RNA itself (Sorefan et al. 2012; Raabe et al. 2014) since DE predictions are only considered reliable when the variability between replicates is lower than the differences between the treatments. In the Normalization component of the pipeline, we incorporate several existing methods for normalization (scalingbased, rank-based, and statistical), with additional features, adapted for sRNA data sets. Scaling normalizations, based on the 
identification of a scaling factor which brings the total number of reads to an a priori fixed total include: the reads per million (RPM)/reads per total (RPT) method (Mortazavi et al. 2008) for which the total abundance of all reads in a sample is considered, upper quartile normalization (Bullard et al. 2010) for which only the reads with abundances in the upper quartile are considered, the trimmed mean of M-values (TMM) (Anders and Huber 2010) and DESeq (Anders and Huber 2010).

Quantile normalization (Bolstad et al. 2003), originally designed for microarray experiments, is also included as an option in the pipeline. This method imposes the same distribution of ranks over all sequences in the data set. We adapted this method to sRNA sequencing data by adding two extra conditions: (i) If, within a sample, two or more reads have the same abundance before normalization, they are assigned the same abundance after normalization which is the average of the normalized abundances. (ii) If a read is not present in the original sample (abundance $=0$ ) then it is assigned an expression level of 0 in its normalized version.

We also include a subsampling-based normalization which is an adapted version of the method described in Li et al. (2012). Our method is based on sampling reads (without replacement) to the minimum library size (for all samples that pass the quality check). It consists of two steps: (i) to ensure that the distribution of abundances are consistent within a sample, the sampling is conducted for decreasing proportions until the sample's distribution has significantly changed or the lowest sample size has been reached; (ii) a subsample of reads with a fixed total is selected repeatedly and, using bootstrapping, the variability of the subsamples is tested. If the variability is low, a random sample (representative for the distribution, i.e., not an outlier) is selected.

\section{Differential expression call}

To identify DE sequences between conditions/treatments, the pipeline includes a confidence interval (CI)-based approach (LopezGomollon et al. 2012; Mohorianu et al. 2013). For each sequence, in each condition, a CI is calculated over replicate expressions using either Chebyshev's intervals calculated from the mean and the standard deviation (Singh et al. 2006) or the minimum and maximum expression levels if only two replicates are available. For a selected comparison between a reference and observed condition, the direction of DE and its amplitude are also calculated. A directional descriptor from the set (up [U], down [D], straight [S]) is assigned to each sequence as follows: $\mathrm{S}$ is used if the CIs overlap, $\mathrm{U}$ indicates that the observed $\mathrm{CI}$ is higher than the reference, and $\mathrm{D}$ indicates the opposite result. The issue of performing pairwise comparisons with sample counts greater than two can then be addressed by forming patterns using the $(U, D, S)$ descriptors. This allows sorting and filtering of sequences that result in potentially relevant/interesting expression changes throughout the course of the experiment.

The amplitude of the difference in expression between conditions is considered on proximate extremes (the closest ends of the neighboring CI) of the reference and observed CIs and is only calculated on sequences that have been assigned a $\mathrm{U}$ or $\mathrm{D}$ descriptor. The amplitude is calculated using the $\log _{2}$ offset fold change (LOFC) method previously described in Mohorianu et al. $(2011,2013)$. The offset prevents low abundance variation from being included in the significant DE distribution. The aim of the offset-approach is to reduce the number of false positives from low abundance sequences and to allow fold change values to be used directly when assessing the relative significance of differentially expressed sequences.

To determine an appropriate offset for a data set, the pipeline can be used to estimate the abundance level around which the majority of noise-related reads lie. Previous studies have observed that low abundance regions/loci have a high strand bias (derived from the reduced number of reads), but loci within the noise-to-signal range have no preferred strand bias (Mohorianu et al. 2011). Based on this observation, the method assigns sRNAs to windows of a set length along the genome reference and the total expression and strand bias is then calculated for each window. For all expression levels, the distribution of strand biases is compared to a random uniform distribution using the Kullback-Leibler (KL) divergence measure (Kullback and Leibler 1951). We define the noise-to-signal threshold (the offset) as the value for which the global minimum of the KL divergence distribution is reached. The distribution is smoothed by a LOESS function (Cleaveland 1979) to prevent expression level outliers from giving a local minimum. Expression levels lower than this threshold tend to have a higher divergence from a uniform strand bias due to a low number of incident reads, and expression levels that are higher than the threshold have an increasing divergence measure due to biologically relevant reads.

\section{DATA DEPOSITION}

The workbench and all the supporting data and tutorials are freely available from http://srna-workbench.cmp.uea.ac.uk. The license is a custom license written for the UEA sRNA Workbench and can be found in the Workbench installation directory or by visiting http://srna-workbench.cmp.uea.ac.uk/wp-content/uploads/2016/

11/sRNA-WorkbenchEULA.pdf. There are no restrictions on use other than requiring citations to specific papers when conducting research with the software; specific details can be found on the website.

\section{SUPPLEMENTAL MATERIAL}

Supplemental material is available for this article.

\section{ACKNOWLEDGMENTS}

The authors thank the members of the Dalmay and Moulton laboratories for constructive discussions and suggestions. This study was supported by the Biotechnology and Biological Sciences Research Council, grant numbers BB/L003139/1, BB/L021269/1.

Received September 23, 2016; accepted February 28, 2017.

\section{REFERENCES}

Anders S, Huber W. 2010. Differential expression analysis for sequence count data. Genome Biol 11: R106.

Anders S, McCarthy DJ, Chen Y, Okoniewski M, Smyth GK, Huber W, Robinson MD. 2013. Count-based differential expression analysis of RNA sequencing data using $\mathrm{R}$ and Bioconductor. Nat Protoc 8: $1765-1786$.

Axtell MJ. 2013. ShortStack: comprehensive annotation and quantification of small RNA genes. RNA 19: 740-751.

Barquist L, Vogel J. 2015. Accelerating discovery and functional analysis of small RNAs with new technologies. Annu Rev Genet 49: 367-394. 
Barrett T, Wilhite SE, Ledoux P, Evangelista C, Kim IF, Tomashevsky M, Marshall KA, Phillippy KH, Sherman PM, Holko M, et al. 2013. NCBI GEO: archive for functional genomics data sets-update. Nucleic Acids Res 41: D991-D995.

Bartel DP. 2009. MicroRNAs: target recognition and regulatory functions. Cell 136: 215-233.

Bland JM, Altman DG. 1986. Statistical methods for assessing agreement between two methods of clinical measurement. Lancet 1: 307-310.

Bolstad BM, Irizarry RA, Astrand M, Speed TP. 2003. A comparison of normalization methods for high density oligonucleotide array data based on variance and bias. Bioinformatics 19: 185-193.

Brodersen P, Voinnet O. 2006. The diversity of RNA silencing pathways in plants. Trends Genet 22: 268-280.

Bullard JH, Purdom E, Hansen KD, Dudoit S. 2010. Evaluation of statistical methods for normalization and differential expression in mRNA-seq experiments. BMC Bioinformatics 11: 94.

Camps C, Saini HK, Mole DR, Choudhry H, Reczko M, GuerraAssunção JA, Tian YM, Buffa FM, Harris AL, Hatzigeorgiou AG, et al. 2014. Integrated analysis of microRNA and mRNA expression and association with HIF binding reveals the complexity of microRNA expression regulation under hypoxia. Mol Cancer 13: 28.

Carthew RW, Sontheimer EJ. 2009. Origins and mechanisms of miRNAs and siRNAs. Cell 136: 642-655.

Chen X. 2012. Small RNAs in development-insights from plants. Curr Opin Genet Dev 22: 361-367.

Cleaveland WS. 1979. Robust locally weighted regression and smoothing scatterplots. J Am Stat Assoc 74: 829-836.

Davis MP, van Dongen S, Abreu-Goodger C, Bartonicek N, Enright AJ. 2013. Kraken: a set of tools for quality control and analysis of highthroughput sequence data. Methods 63: 41-49.

Dillies MA, Rau A, Aubert J, Hennequet-Antier C, Jeanmougin M, Servant N, Keime C, Marot G, Castel D, Estelle J, et al. 2013. A comprehensive evaluation of normalization methods for Illumina highthroughput RNA sequencing data analysis. Brief Bioinform 14: 671-683.

Donaszi-Ivanov A, Mohorianu I, Dalmay T, Powell PP. 2013. Small RNA analysis in Sindbis virus infected human HEK293 cells. PLoS One 8: e84070.

Fasold M, Langenberger D, Binder H, Stadler PF, Hoffmann S. 2011. DARIO: a ncRNA detection and analysis tool for next-generation sequencing experiments. Nucleic Acids Res 39: W112-W117.

Garber M, Grabherr MG, Guttman M, Trapnell C. 2011. Computational methods for transcriptome annotation and quantification using RNA-seq. Nat Methods 8: 469-477.

Gupta V, Markmann K, Pedersen CN, Stougaard J, Andersen SU. 2012. shortran: a pipeline for small RNA-seq data analysis. Bioinformatics 28: $2698-2700$.

Habegger L, Sboner A, Gianoulis TA, Rozowsky J, Agarwal A, Snyder M, Gerstein M. 2011. RSEQtools: a modular framework to analyze RNA-Seq data using compact, anonymized data summaries. Bioinformatics 27: 281-283.

Hardcastle TJ, Kelly KA. 2010. baySeq: empirical Bayesian methods for identifying differential expression in sequence count data. $B M C$ Bioinformatics 11: 422 .

Jaccard P. 1901. Etude comparative de la distribution forale dans une portion des Alpes et de Jura. Bull Soc Vaudoise Sci Nat 37: 547-579.

Kayala MA, Baldi P. 2012. Cyber-T web server: differential analysis of high-throughput data. Nucleic Acids Res 40: W553-W559

Kullback S, Leibler RA. 1951. On information and sufficiency. Ann Math Stat 22: 79-86.

Li J, Witten DM, Johnstone IM, Tibshirani R. 2012. Normalization, testing, and false discovery rate estimation for RNA-sequencing data. Biostatistics 13: 523-538.

Lippman Z, Martienssen R. 2004. The role of RNA interference in heterochromatic silencing. Nature 431: 364-370.

Lohse M, Bolger AM, Nagel A, Fernie AR, Lunn JE, Stitt M, Usadel B. 2012. RobiNA: a user-friendly, integrated software solution for RNA-seq-based transcriptomics. Nucleic Acids Res 40: W622W627.

Lopez-Gomollon S, Mohorianu I, Szittya G, Moulton V, Dalmay T. 2012. Diverse correlation patterns between microRNAs and their targets during tomato fruit development indicates different modes of microRNA actions. Planta 236: 1875-1887.

Love MI, Huber W, Anders S. 2014. Moderated estimation of fold change and dispersion for RNA-seq data with DESeq2. Genome Biol 15: 550 .

Mapleson D, Mohorianu I, Pais H, Stocks MB, Folkes L, Moulton V. 2014. Processing large-scale small RNA datasets in silico. In Nextgeneration sequencing: current technologies and applications. Caister Academic Press, UK.

McCormick KP, Willmann MR, Meyers BC. 2011. Experimental design, preprocessing, normalization and differential expression analysis of small RNA sequencing experiments. Silence 2: 2 .

Meister G. 2013. Argonaute proteins: functional insights and emerging roles. Nat Rev Genet 14: 447-459.

Mohorianu I, Moulton V. 2010. Revealing biological information using data structuring and automated learning. Recent Pat DNA Gene Seq 4: 181-191.

Mohorianu I, Schwach F, Jing R, Lopez-Gomollon S, Moxon S, Szittya G, Sorefan K, Moulton V, Dalmay T. 2011. Profiling of short RNAs during fleshy fruit development reveals stage-specific sRNAome expression patterns. Plant J 67: 232-246.

Mohorianu I, Stocks MB, Wood J, Dalmay T, Moulton V. 2013. CoLIde: a bioinformatics tool for CO-expression-based small RNA loci identification using high-throughput sequencing data. RNA Biol 10: 1221-1230.

Molnar A, Schwach F, Studholme DJ, Thuenemann EC, Baulcombe DC. 2007. miRNAs control gene expression in the single-cell alga Chlamydomonas reinhardtii. Nature 447: 1126-1129.

Morey JS, Ryan JC, Van Dolah FM. 2006. Microarray validation: factors influencing correlation between oligonucleotide microarrays and real-time PCR. Biol Proc Online 8: 175-193.

Mortazavi A, Williams BA, McCue K, Schaeffer L, Wold B. 2008. Mapping and quantifying mammalian transcriptomes by RNAseq. Nat Methods 5: 621-628.

Moxon S, Schwach F, Dalmay T, Maclean D, Studholme DJ, Moulton V. 2008. A toolkit for analysing large-scale plant small RNA datasets. Bioinformatics 24: 2252-2253.

Müller S, Rycak L, Winter P, Kahl G, Koch I, Rotter B. 2013. omiRas: a Web server for differential expression analysis of miRNAs derived from small RNA-Seq data. Bioinformatics 29: 2651-2652.

Omidvar V, Mohorianu I, Dalmay T, Fellner M. 2015. Identification of miRNAs with potential roles in regulation of anther development and male-sterility in 7B-1 male-sterile tomato mutant. BMC Genomics 16: 878

Ozsolak F, Milos PM. 2011. RNA sequencing: advances, challenges and opportunities. Nat Rev Genet 12: 87-98.

Raabe CA, Tang TH, Brosius J, Rozhdestvensky TS. 2014. Biases in small RNA deep sequencing data. Nucleic Acids Res 42: 1414-1426.

Rajagopalan R, Vaucheret H, Trejo J, Bartel DP. 2006. A diverse and evolutionarily fluid set of microRNAs in Arabidopsis thaliana. Genes Dev 20: 3407-3425.

Rapaport F, Khanin R, Liang Y, Pirun M, Krek A, Zumbo P, Mason CE, Socci ND, Betel D. 2013. Comprehensive evaluation of differential gene expression analysis methods for RNA-seq data. Genome Biol 14: R95.

Rueda A, Barturen G, Lebron R, Gomez-Martín C, Alganza A, Oliver JL, Hackenberg M. 2015. sRNAtoolbox: an integrated collection of small RNA research tools. Nucleic Acids Res 43: W467-W473.

Sadd BM, Barribeau SM, Bloch G, de Graaf DC, Dearden P, Elsik CG, Gadau J, Grimmelikhuijzen CJ, Hasselmann M, Lozier JD, et al. 2015. The genomes of two key bumblebee species with primitive eusocial organization. Genome Biol 16: 76.

SEQC/MAQC-III Consortium. 2014. A comprehensive assessment of RNA-seq accuracy, reproducibility and information content by the 
Sequencing Quality Control Consortium. Nat Biotechnol 32: 903-914.

Singh A, Maichle R, Lee S. 2006. On the computation of 95\% upper confidence limit of the unknown population mean based upon data sets with below detection limit observations. US Environmental Protection Agency, Office of Research and Development.

Soneson C, Delorenzi M. 2013. A comparison of methods for differential expression analysis of RNA-seq data. BMC Bioinformatics 14: 91.

Sorefan K, Pais H, Hall AE, Kozomara A, Griffiths-Jones S, Moulton V, Dalmay T. 2012. Reducing ligation bias of small RNAs in libraries for next generation sequencing. Silence 3: 4 .

Stocks MB, Moxon S, Mapleson D, Woolfenden HC, Mohorianu I, Folkes L, Schwach F, Dalmay T, Moulton V. 2012. The UEA sRNA workbench: a suite of tools for analysing and visualizing next generation sequencing microRNA and small RNA datasets. Bioinformatics 28: 2059-2061.

Studholme DJ. 2012. Deep sequencing of small RNAs in plants: applied bioinformatics. Brief Funct Genomics 11: 71-85.

Sun J, Nishiyama T, Shimizu K, Kadota K. 2013. TCC: an R package for comparing tag count data with robust normalization strategies. $B M C$ Bioinformatics 14: 219.

Szittya G, Moxon S, Pantaleo V, Toth G, Rusholme Pilcher RL, Moulton V, Burgyan J, Dalmay T. 2010. Structural and functional analysis of viral siRNAs. PLoS Pathog 6: e1000838.
Vidal EA, Moyano TC, Krouk G, Katari MS, Tanurdzic M, McCombie WR, Coruzzi GM, Gutierrez RA. 2013. Integrated RNA-seq and sRNA-seq analysis identifies novel nitrate-responsive genes in Arabidopsis thaliana roots. BMC Genomics 14: 701.

Voinnet O. 2009. Origin, biogenesis, and activity of plant microRNAs. Cell 136: 669-687.

Xu P, Mohorianu I, Yang L, Zhao H, Gao Z, Dalmay T. 2014. Small RNA profile in moso bamboo root and leaf obtained by high definition adapters. PLoS One 9: e103590.

Yang YH, Dudoit S, Luu P, Lin DM, Peng V, Ngai J, Speed TP. 2002. Normalization for cDNA microarray data: a robust composite method addressing single and multiple slide systematic variation. Nucleic Acids Res 30: e15.

Zhai J, Zhao Y, Simon SA, Huang S, Petsch K, Arikit S, Pillay M, Ji L, Xie M, Cao X, et al. 2013. Plant microRNAs display differential $3^{\prime}$ truncation and tailing modifications that are ARGONAUTE1 dependent and conserved across species. Plant Cell 25: 2417-2428.

Zhou X, Lindsay H, Robinson MD. 2014. Robustly detecting differential expression in RNA sequencing data using observation weights. Nucleic Acids Res 42: e91.

Zhu H, Zhou Y, Castillo-González C, Lu A, Ge C, Zhao YT, Duan L, Li Z, Axtell MJ, Wang XJ, et al. 2013. Bidirectional processing of pri-miRNAs with branched terminal loops by Arabidopsis Dicerlike1. Nat Struct Mol Biol 20: 1106-1115. 

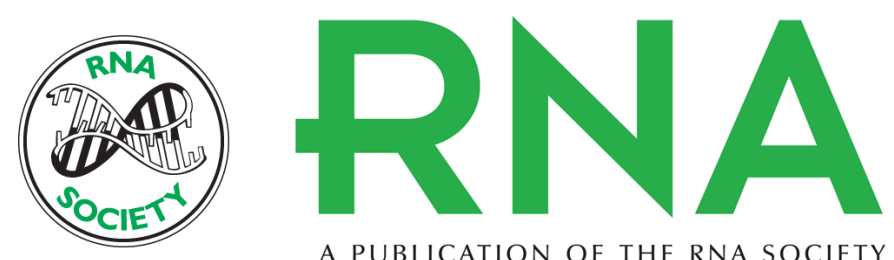

A PUBLICATION OF THE RNA SOCIETY

\section{Comprehensive processing of high-throughput small RNA sequencing data including quality checking, normalization, and differential expression analysis using the UEA sRNA Workbench}

Matthew Beckers, Irina Mohorianu, Matthew Stocks, et al.

RNA 2017 23: 823-835 originally published online March 13, 2017

Access the most recent version at doi:10.1261/rna.059360.116

Supplemental Material

References

Open Access

Creative Commons License

Email Alerting Service
http://rnajournal.cshlp.org/content/suppl/2017/03/13/rna.059360.116.DC1

This article cites 61 articles, 3 of which can be accessed free at: http://rnajournal.cshlp.org/content/23/6/823.full.html\#ref-list-1

Freely available online through the RNA Open Access option.

This article, published in $R N A$, is available under a Creative Commons License (Attribution 4.0 International), as described at http://creativecommons.org/licenses/by/4.0/.

Receive free email alerts when new articles cite this article - sign up in the box at the top right corner of the article or click here.

To subscribe to RNA go to:

http://rnajournal.cshlp.org/subscriptions 